\title{
Effects of a 4-week intervention with ready-made bioactive enriched pancakes on biomarkers of the metabolic syndrome
}

\author{
S. Sutulic ${ }^{1}$, C. Bösch ${ }^{1}$, L. Marshall ${ }^{1}$, A. Bordoni ${ }^{2}$, H. Hingyi ${ }^{3}$, M. Muller ${ }^{4}$, C. Orfila ${ }^{1}$ and on \\ behalf of the PATHWAY-27 consortium Grant agreement 311876 \\ ${ }^{1}$ School of Food Science and Nutrition, University of Leeds, Leeds LS2 9JT, ${ }^{2}$ Dipartimento di Scienze e Tecnologie \\ Agro-Alimentari, Università di Bologna, Italy, ${ }^{3}$ Adexgo Kft., Hungary and ${ }^{4}$ AdWare Research Kft., Hungary
}

In the UK it is estimated that 1 in 4 adults are at greater risk of heart disease and stroke due to presence of the metabolic syndrome $(\mathrm{MetS})^{(1)}$. The MetS is a term used to describe any combination of at least three of the following conditions: high blood pressure, increased waist circumference, impaired fasting glucose, increased fasting triglycerides, and low HDL cholesterol.

Components of foods that may improve conditions associated with MetS include the omega-3 fatty acid docosahexaenoic acid (DHA) and the soluble plant fibre beta-glucan (BG), which are considered beneficial for maintaining normal blood triglyceride and cholesterol concentrations ${ }^{(2,3)}$. There is also clinical evidence that anthocyanins (AC) may improve HDL cholesterol in adults with dyslipidaemia ${ }^{(4)}$.

In a double-blind, parallel-arm, randomised pilot study, 35 participants $(\mathrm{M}=40 \%)$, mean age $45 \mathrm{y}$, with or at risk of MetS were recruited to consume ready-made pancakes enriched with DHA, BG, or AC alone or in combinations of DHA + BG or DHA + AC every day for 4 weeks. At baseline and endpoint markers of MetS were measured. Mean change, from baseline to endpoint, in biomarkers of the MetS are summarised in Table 1.

Table 1: Mean change in biomarkers of the MetS. HDL-C, HDL cholesterol; SBP, systolic blood pressure; DBP, diastolic blood pressure; SD, standard deviation. Results were not statistically significant.

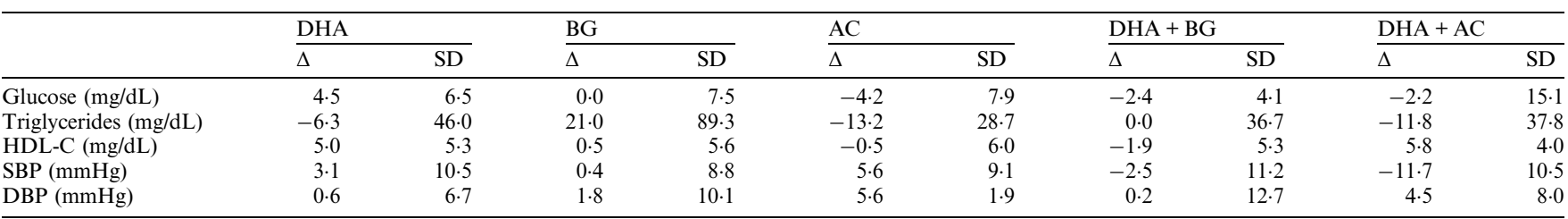

The objective of this pilot study was to select the enrichment type most effective at improving fasting blood triglycerides and/or HDL cholesterol, to be consumed in the subsequent PATHWAY-27 12-week placebo-controlled, multi-centre trial. Pancakes enriched with DHA and AC alone and in combination appear to be the most effective. DHA + AC enrichment was selected for further study.

1. Department of Health (2014) NHS Choices: Metabolic syndrome, http://www.nhs.uk/conditions/metabolic-syndrome/Pages/Introduction.aspx, (accessed January 2016).

2. EFSA Panel on Dietetic Products, Nutrition and Allergies (2010) EFSA Journal 8, (10), 1734

3. EFSA Panel on Dietetic Products, Nutrition and Allergies (2009) EFSA Journal 7, (9), 1254.

4. Qin et al. (2009) Am J Clin Nutr 90, 485-492. 